\title{
Face-ism and military leadership
}

\author{
Xyle $\mathrm{Ku}^{*} \cdot$ Seungju Hyun ${ }^{* *} \cdot$ Jaewon $\mathrm{Ko}^{* * *}$
}

\begin{abstract}
Deriving inferences from facial appearance is called face-ism. In particular, people make rapid and accurate inferences about the targets' character based on their appearance. Over the last 15 years, the effects of a leader's facial appearance have been vigorously studied in the domain of psychology and leadership research worldwide. Previous studies suggest that facial appearance significantly predicts leader selection, thus leading more competent, dominant, trustworthy, and attractive individuals to be selected as leaders. Further, they indicate that this relationship depends on the circumstances (i.e., wartime and time of peace). The current study reviewed recent research on face-ism and leadership, while discussing the factors of the face effects, the main method, findings, limitations, and future directions of the research. Finally, the authors discussed the implications of face-ism in the field of military leadership.
\end{abstract}

Keywords : face-ism, leadership, leader selection, military leadership

(a) (1) This work is licensed under a Creative Commons Attribution 4.0 International License.

* (First Author) Korea Military Academy, Department of Psychology, Assistant Professor, xyleku@gmail.com

** (Co-Author) Korea Military Academy, Department of Psychology, Instructor, tmdwn5997@gmail.com

*** (Corresponding Author) Korea Military Academy, Department of Psychology, Professor, wonko64@gmail.com 


\section{I. 서론}

1960년 9월 26일은 미국 역사상 최초로 대통령 후보자 토론회가 TV로 중계된 날로 기록된다.1) 당시 대통령 후보자는 리처드 닉슨(Richard M. Nixon)과 존 케네디(John F. Kennedy)였다. 시장 조사 회사인 Sindlinger \& Company의 통계자료에 따르면, 대통령 토론회를 라디오로 들은 사람들 은 토론의 승리자로 닉슨을 선택했으나, TV로 시청한 사람들은 케네디를 선택하는 상반된 결과가 나타났다(Gidlow, 2014).2) 당시, 닉슨은 케네디보다 사회 이슈에 대해 더 많은 경험과 지식이 있음 에도 불구하고, TV에서 보인 그의 외모는 창백하고 아파 보이며 단정하지 못한 데 반해, 케네디는 젊고 건강하며 침착해 보인 것으로 평가받았다(Gidlow, 2014). 결국, 케네디는 닉슨과의 경쟁에서 승리하며 대통령으로 당선되었다. 이는 리더 선택 과정에서 외모의 중요성을 나타내는 대표적인 사 례로 여겨진다(Little, 2014; Gomulya et al., 2017).

리더십 연구의 주요 주제가 리더 선택이라는 점을 고려했을 때(Vardiman et al., 2006), 리더십 연구에 있어서 어떤 리더 외모가 선호되는지, 그리고 특정 외모가 어떤 조건과 환경에서 선호되거 나 혹은 되지 않는지 연구될 필요성을 시사한다. 최근 약 15 년 동안, 심리학 및 리더십 연구 분야에 서 리더 외모(face 혹은 facial appearance)3)에 관한 연구가 활발히 진행 중이다. 특히, 미국 상 - 하 원 의원 선거에 출마한 후보자들 중, 당선자와 차점자(runner-up)에 대한 외모 평가값이 실제 선거 결과를 예측함을 밝힌 논문이 저명 학술지에 게재되면서(Todorov et al., 2005), 리더 외모 연구가 본격적으로 증대되었다. 특히, 주로 어떠한 외모적 특성을 지닌 사람들이 리더로 선호되거나 선발 되는가(리더 선택)에 초점을 둔 연구가 많이 진행되어 왔다(Antonakis \& Eubanks, 2017; Poutvaara, 2014).4)

연구 결과를 종합하면, 유능하고, 지배적이고, 신뢰롭고, 매력적인 외모적 특성을 지닌 사람들이 그렇지 않은 사람들에 비해 리더로 선택되거나 선호되는 경향성이 강했으며, 특히 리더를 선택하는 상황적 맥락이 리더의 외모적 특성과 리더 선택의 관계에 영향을 미치는 것으로 나타났다. 따라서 본 연구는 리더 외모와 리더 선택(선호도)의 관계를 밝힌 선행연구들에 대한 고찰을 바탕으로 군 리더십 분야에서 리더 외모 연구가 가질 수 있는 함의점을 탐색적으로 논의하는 데 목적을 두고 실시되었다. 우선, 리더 외모 효과가 나타나는 이유에 관해 논의하고 선행연구들이 사용한 구체적 인 연구 방법, 연구 결과, 한계점 및 향후 연구방향을 체계적으로 제시했다. 끝으로 결론 및 제언

1) 이 날, 사람들은 대통령 후보 토론회를 라디오로 듣거나 TV로 시청했다. 그 이전까지는 대통령 토론회를 라디오를 통해서만 들을 수 있었다.

2) Sindlinger \& Company의 통계자료는 통계적인 오류가 많은 것으로 지적되었으나 이후, 사회과학 기반의 연구를 통해 같은 결과가 나타나는 것으로 확인되었다(Druckman, 2003).

3) 연구자들은 face와 facial appearance의 용어를 혼용하고 있다.

4) 리더 선택 이외에 리더 성공 혹은 효과성이 리더 외모와 어떻게 연관되는가에 관한 연구도 다수 진행되어 왔으나, 본 연구에서는 리더 선택에 초점을 두고 논의를 진행하였다. 
부분에서는, 군 리더십 분야에서 리더 외모 연구가 가질 수 있는 함의점을 전 - 평시 선호되는 리더 외모의 차이 및 군 인사에서 외모가 미칠 수 있는 영향을 중심으로 논의했다.

\section{ㅍ. 페이시즘과 그 효과의 이유}

‘누구를 리더로 선택할 것인가’는 인류 역사에서 가장 중요한 선택 중 하나로 여겨진다(Van Vugt \& Grabo, 2015). 그럼에도 불구하고, 다수의 연구들이 리더 후보자들의 외모적 특성이 사람들 의 리더 선택 과정에 유의한 영향을 미치는 현상을 보고하고 있다(Bastardoz \& Van Vugt, 2019; Poutvaara, 2014; Van Vugt \& Grabo, 2015).5) 사람들은 리더 후보자들의 외모를 통해 신속하게 누 가 가장 리더다운지 혹은 누가 가장 리더로 적합한지에 관한 판단을 내린다는 것이다. 왜 이러한 현상이 나타나는 것일까?

사람들은 일반적으로 대상의 외모를 대상의 성격을 알려주는 중요한 창구로 인식하며(Hassin \& Trope, 2000; Todorov et al., 2015), 대상의 특성 평가 시 외모의 영향을 많이 받는다(Sacco \& Brown, 2018). 예를 들어, 사람들은 대상의 성격(Little \& Perrett, 2007), 나이(George \& Hole, 2000), 사회 계층(Bjornsdottir \& Rule, 2017), 경계선 성격 장애(Daros et al., 2016), 성적 지향성 (Rule \& Alaei, 2016) 그리고 유아의 성별(Tskhay \& Rule, 2016) 등을 외모만을 통해 정확하게 인 식한다. Little과 Perrett(2007)의 연구에서 피험자들에게 특정한 특성(외향성, 성실성 등)을 나타내 는 합성 사진(composite image)을 제시하고 사진 속 대상의 성격을 추측하게 했는데, 우연 수준 이 상으로 합성 사진이 나타내는 외향성과 성실성의 특성(성격)을 정확하게 맞추는 것으로 나타났다.

이렇게 사람들이 대상의 외모를 통해 대상의 특성을 추론하는 경향성을 '페이시즘(face-ism)'이 라 한다(Olivola et al., 2014). Willis와 Todorov(2006)는 어떤 대상의 사진을 0.1초 동안 제시했을 때 피험자들이 사진으로부터 평가한 특성(유능함, 신뢰성, 공격성 등)값과 시간제한 없이 사진을 제 시했을 때 사진으로부터 평가한 특성 값이 매우 높은 상관관계를 보임을 밝혔다. 후속 연구에 따르 면, 외모로부터 대상의 특성을 추론하는 것은 0.033초면 충분한 것으로 밝혀졌다(Todorov et al., 2009). 이처럼 페이시즘 현상은 외모만을 통해, 빠르고 비교적 정확하게 대상의 특성을 평가하는 사 람들의 경향성을 나타낸다.

페이시즘은 학습에 의한 결과라기보다는 인간의 선천적이고 보편적인 능력인 것으로 보인다 (Antonakis \& Dalgas, 2009; Cogsdill et al., 2014), 2002년 프랑스 의회 선거에 출마한 후보자들의

5) 리더 외모는 리더 선택 시 사용되는 추단 중 하나이며, 리더의 성별, 키, 목소리 등 다양한 비언어적 요소들도 리더 선택 과정에 영향을 미치는 것으로 나타났다(Van Vugt \& Ahuja, 2010). 예를 들어, 키가 클수록(Elgar, 2016; Lindqvist, 2012), 저음의 목소리를 가질수록(Anderson \& Klofstad, 2012; Klofstad et al., 2012; Tigue et al., 2012). 리더가 될 가능성이 높은 것으로 나타난다. 본 연구에서는 다른 비언어적 요소들에 대한 논의는 제외하고, 리더의 외모에 중점을 두고 연구를 진행하였다. 
사진을 사용한 Antonakis와 Dalgas(2008)의 연구가 이를 뒷받침한다. 연구자들은 피험자들에게 사 진 속 후보자들의 유능함의 정도를 외모만을 통해 평가하도록 했는데, 5 세에서 13 세 사이의 어린이 들과 어른들의 평가가 통계적으로 차이가 나지 않았으며, 평가값이 선거 결과를 유의하게 예측했 다. 또한, 대상의 외모를 통해 특정한 특성을 추론하는 경향성은 응답자들이 동양 문화권에 속하는 지 아니면 서양 문화권에 속하는지에 따른 영향도 받지 않는 것으로 나타난다. 예를 들어, Rule과 그의 동료들(2010)은 일본인과 미국인 피험자들에게 일본 및 미국 정치인들의 사진을 제시하고 그 들의 외모적 지배성, 성숙성, 호감도, 신뢰성 등의 특성에 대해 평가하게 하였는데, 일본인과 미국 인들의 평가값은 통계적으로 차이가 나지 않았다.

Van Vugt와 Grabo(2015)는 페이시즘의 경향성을 진화 수반성 가설(Evolutionary-Contingency Hypothesis)로 설명한다. 이들은 인류의 진화 과정에서 누구를 리더로 선발하고 따를 것인가는 개 인과 집단의 생존과 직결되는 중요한 선택이었을 것으로 가정한다. 그리고 이러한 선택의 기반이 되었던 것이 외모와 같은 비언어적 단서였을 것으로 추정한다(Bastardoz \& Van Vugt, 2019; Grabo \& Van Vugt, 2018). 그 이유는 이러한 비언어적 단서 외에는 리더로서의 자질을 평가하는 데 근거로 삼을 만한 합리적 단서들이 부족했기 때문이다(Van Vugt \& Ahuja, 2010). 따라서 먼 과거6)에는 외모를 포함한 비언어적 단서들이 리더 선택에 있어 중요한 단서로 사용되었을 것이며, 이러한 비언어적 단서를 사용하는 경향성이 그 효용성과 관계없이 지금도 남아 있는 것으로 평가 한다(Grabo \& Van Vugt, 2018; Poutvaara, 2014). 사람들에게는 특정한 외모적 특성을 지닌 사람 에 대해 '리더다운(leader-like)' 사람이라고 판단하는 심리 과정이 내재되어 있으며(Antonakis \& Eubanks, 2017; Bastardoz \& Van Vugt, 2019; Little \& Roberts, 2012; Petersen, \& Aarøe, 2012; Re \& Rule, 2015; 2017; Van Vugt \& Grabo, 2015), 이는 '가장 매력적인 혹은 가장 유능해 보이는 외모를 가진 사람을 찾아서 따라라'와 같이 추단을 사용하는 형태로 나타나는 것으로 여겨진다 (Spisak et al., 2014).

\section{III. 주요 연구방식}

연구 방식은 대체로 다음과 같다. 우선 연구자들은 연구 가설에 따라 피험자들에게 다양한 리더 사진들을 제시한다. 이후, 피험자들에게 외모만을 통해 대상의 여러 특성들을 추론하여 평가하게 한다. 그리고 피험자들의 리더 외모 평정치를 연구 목적에 따라 대상에 대한 선호도, 실제 리더의 지위, 리더 선발 여부, 선발 시 득표 비율 혹은 득표 수(정치인과 같이 투표를 통해 리더로 선발이 되는 경우)와의 관계를 분석한다. 연구에 따라, 피험자들의 평정치와 가상의 투표에 대한 의도와의

6) 먼 과거는 비교적 소규모의 유전적으로 연계된 사람들이 종족을 이루어 생활했던 진화적 적응의 환경(environmental of evolutionary adaptation, EEA)을 나타낸다(Forsyth, 2018). 
관계를 분석하기도 한다.

\section{1 자극: 리더 사진}

리더 사진의 경우, 주로 특정 국가에서 특정 연도에 실시된 대통령, 주지사, 상 - 하원 의원, 국회 의원 선거 등 정치적 선거에 출마한 후보자들의 실제 사진이 사용되는 경우가 가장 많다(한국: $\mathrm{Na}$ et al., 2015; 독일: Rosar et al., 2008; 미국: Ballew \& Todorov, 2007; Olivola \& Todorov, 2010; Rule et al., 2010; Todorov et al., 2005; 불가리아: Sussman et al., 2013; 브라질과 멕시코: Lawson et al., 2010; 일본: Rule et al., 2010; 스위스: Lutz, 2010; 아일랜드: Buckley et al., 2007; 영국: Banducci et al., 2008; 이탈리아: Castelli et al., 2009; 캐나다: Rule \& Ambady, 2010; 프랑스: Antonakis \& Dalgas, 2009; 핀란드: Berggren et al., 2010; Poutvaara, Jordahl, \& Berggren, 2009).7)

연구의 목적상 실제 사진이 아닌 특정한 소프트웨어를 통해 제작한 얼굴의 이미지를 제시하는 경우도 있다(Little, 2014; Little et al., 2012; Re et al., 2013; Spisak, Dekker et al., 2012; Spisak, Homan et al. 2012). 예를 들어, Little과 그의 동료들(2007)은 조지 부시(George Bush)와 존 케리 (John Kerry) 사진을 중성 얼굴 이미지로 변환하여 연구에 사용한 바 있다.

\section{2 리더 사진의 변경 및 통제}

실제 리더 사진들이 연구에 사용되는 경우, 사진 자극 간의 질적 차이를 최소화한다. 예를 들어, 사진의 밝기, 배경 및 사진 속 대상과 배경의 비율을 동일하게 맞추며, 색상은 회색음영으로 수정하 여 사용한다. 사진은 무작위 순서로 제시되며, 2 명의 사진이 같이 제시되는 경우에는 좌 - 우가 무 작위로 바뀌어 제시된다. 사진 속의 대상은 외모 평가에 영향을 미칠 수 있는 다양한 얼굴 표정과 정서 표현을 하고 있기 때문에(Trichas et al., 2017), 별도의 피험자들에게 사진 속 대상의 정서 표 현에 관해 응답하도록 하고, 이 평정치를 분석 간 통제하는 경우가 많다(Rule \& Ambady, 2008; 2009; Re \& Rule, 2016a). 예를 들어, Re와 Rule(2016a)은 사진 속 리더들의 정서 표현을 7점 척도 ( $1=$ 중성적인, $4=$ 행복한, $7=$ =매우 행복한)로 응답하게 하였으며, 이 평정치를 분석 간 통제하였다.

7) 정치인들의 사진이 많이 사용되는 이유는, 다른 분야에 비해 정치적 선거 과정에서 유권자들의 투표 행위에 영향을 주는 변수 중 하나로 리더의 외모가 많이 제시되기 때문인 것으로 보인다(Little \& Roberts, 2012). 또한, 공공 기관 및 기업과 같은 대부분의 집단에서 상향식의 리더 선발(하급자가 리더를 선발) 방식보다 하향식의 리더 선발(상부에서 리더를 선발) 방식을 도입하고 있다는 점도 그 이유로 제시될 수 있다(Van Vugt \& Ahuja, 2010). 


\section{3 사진 평정 방식}

연구자들은 피험자들에게 사진만 제시하고, 일반적으로 사진 속 대상의 신분, 연령 등 대상과 관 련된 어떠한 정보도 알려주지 않는다. 피험자들은 대상의 외모만을 통해 대상의 특성을 추론하여 평가한다. 평가하는 특성은 주로 대상의 유능함, 지배성, 신뢰성 그리고 매력 등이다(Van Vugt, \& Grabo, 2015). 이 외에도, 연구 목적과 가설에 따라, 어리게 보이는 얼굴(Livingston \& Pearce, 2009), 얼굴의 대칭성(Senior et al., 2012), 얼굴의 너비 대 높이 비율(Alrajih \& Ward, 2014) 등이 평가되기도 한다.

질문의 방식은 크게 2가지로 구분된다(Stoker et al., 2016). 첫 번째 방식은 2 명의 사진을 동시에 보여준 후, 2 명 중 어떤 대상이 특정한 특성을 더 가지고 있는 사람으로 보이는지를 묻는 방식이다 (Todorov et al., 2005). 예를 들어, Todorov와 그의 동료들(2005)은 " 2 명 중 누가 더 유능한 사람으 로 보입니까?라는 질문을 사용했다. 두 번째 방식은 1 명의 리더 사진을 제시하고, 그 대상이 특정 한 특성을 얼마나 가지고 있는 사람으로 보이는지에 대해 묻는 방식이다. 예를 들어, Rule과 그의 동료들(2010)은 피험자들에게 '사진 속 대상이 얼마나 유능한 사람으로 보이는가?'라는 질문에 7점 척도 상에서 응답하도록 하였다. 연구 목적에 따라, 직접적인 리더십 능력을 평가하게 하는 연구들 도 있다(Nana et al., 2010; Rule \& Ambady, 2008). 예를 들어, Rule과 Ambady(2008)는 피험자들에 게 사진 속의 대상에 대해 ‘이 사람이 얼마나 회사를 잘 이끄는 사람일 것 같습니까?'라는 질문에 응답하도록 했다.

1 명의 리더 사진에 대해 여러 특성들을 질문하는 경우, 이 질문들은 무작위 순서로 제시되며, 연 구에 사용되는 사진의 갯수가 많은 경우에는 피험자의 피로도를 고려하여 피험자들마다 무작위로 일부의 사진만 제시하기도 한다. 예를 들어, Re와 Rule(2016a)은 100 명의 리더 사진을 사용하였는 데 사진을 50 개씩 나누어 2 개의 버전을 만들어, 피험자들에게 무작위로 2 개의 버전 중 1 개의 버전 을 제시하여 응답하도록 했다.

\section{4 분석 변수: 리더 선택(선호도)}

평정 대상의 당선 여부, 득표 비율 그리고 득표수와 같은 구체적인 선거 결과 지표(Berggren et al., 2010; Sussman et al., 2013; Todorove et al., 2005; White et al., 2003)가 사용되기도 하고, "2명 의 대상 중, 누구에게 투표를 하겠는가?'(Little et al., 2007) 혹은 '사진 속의 대상에게 얼마나 투표 할 용의가 있는가?'(Little, 2014; Little et al., 2007; 2012; Spisak, Dekker et al., 2012; Spisak, Homan et al., 2012) 등과 같이 가상의 투표(선호도) 등의 변수들이 결과변수로 주로 사용된다. 


\section{IV. 주요 연구결과}

앞서 제시한 연구 방법들을 통해 리더 외모와 리더 선택의 관계를 확인한 다양한 연구들이 진행 되어 왔다. 특히, 연구자들은 유능하고, 지배적이고, 신뢰로우며, 매력적인 리더의 외모적 특성에 주 로 초점을 두고 연구를 실시했다(Van Vugt \& Grabo, 2015). 따라서 선행연구들의 주요 연구결과 는 유능함, 지배성, 신뢰성 그리고 매력의 4 가지 차원을 중심으로 제시하였다.

\section{1 유능함(competence)}

외모를 통해 유능하다고 판단되는 사람들은 실제로 선거에 당선되는 경향이 있다(Armstrong et al., 2010; Antonakis \& Dalgas, 2009; Ballew \& Todorov, 2007; Castelli et al., 2009; Olivola \& Todorov, 2010; Na et al., 2015; Poutvaara et al., 2009; Sussman et al., 2013; Todorov et al., 2005). 예를 들어, Sussman과 그의 동료들(2013)은 223명의 피험자들에게 2011년에 실시된 불가리아 대통 령선거에 출마한 후보자 18 명의 사진을 무작위 순서로 보여줬다. 그리고 각 대상에 대해 '이 사람이 얼마나 유능한/지배적인/호감 가는/정직한/청렴한/매력적인 사람으로 보입니까?'라는 질문에 7점 척도(1=전혀 그렇지 않다, $7=$ 매우 그렇다)로 응답하게 하였다. 연구 결과, '유능한' 특성에 대한 평 정치는 실제 후보자의 득표 비율과 정적 상관관계를 보인 반면, 나머지 특성들은 유의한 상관관계 가 나타나지 않았다. 또한, 피험자들의 가상 투표 결과도 실제 투표 결과와 통계적으로 유사한 것으 로 나타났다.

외모적 유능함은 리더 선택(특히, 정치인들의 선거 결과)을 예측하는 여러 외모적 특성 중 가장 예측력이 강한 것으로 평가된다(Olivola \& Todorov, 2010). 그 이유는 유능함의 특성이 정치인들에 게 가장 중요한 특성 중 하나로 인식되기 때문인 것으로 보여진다(Trent et al., 1993). 또한, 공포관 리이론(Solomon et al., 1991)을 바탕으로 실시된 연구에서, 피험자들을 죽음을 부각시키는 조건 (mortality salience condition)과 그렇지 않은 조건에 배정시킨 후, 3가지 형태의 리더에 대한 선호 도를 측정했다. 그 결과, 카리스마적 리더, 관계 지향적 리더, 과업 지향적 리더 중, 유능함의 특성 과 연관되는 과업지향적 리더가 조건에 관계없이 가장 선호되는 리더인 것으로 나타났다(Cohen et al., 2004).8) 이는 사람들은 유능한 리더에 대한 선호도가 일반적으로 높으며, 외모적 유능함도 리더 에 대한 선호도에 영향을 미칠 수 있음을 시사한다.

유능해 보이는 외모는 ‘외모로 추정된 나이'와 연관되어 있는 것으로 평가되는데, 이유는 나이는 지혜와 연관된다는 믿음이 있기 때문이다(Van Vugt \& Grabo, 2015). 실제로, 나이 들어 보이는 사 람들이 더 유능하고 리더다운 사람으로 인식되는 것으로 나타났다(Spisak et al., 2014). 또한, 외모

8) 죽음 부각 조건에서는 카리스마적 리더에 대한 선호도가 높아지고, 관계 지향적 리더에 대한 선호도는 낮아지는 것으로 나타났다. 
적 유능함이 리더 선택에 미치는 효과는 해당 국가의 안보 상황에 따라 달라질 수 있는 것으로 제 시되었는데 예를 들어, 안보 위협이 낮은 국가보다 높은 국가에서 유능해 보이는 외모를 가진 후보 자들이 대통령으로 선출되는 경향성이 더 강하게 나타났으며, 외모적 유능함과 정치적 선거에서의 득표 비율의 상관관계도 안보 위협이 높은 국가에서만 유의한 것으로 나타났다(Ku, 2019).

\section{2 지배성(dominance)}

외모적 지배성과 리더 선호도의 관계는 상황에 따라 다르게 나타나는 것이 특징이다(Little, 2014; Little et al., 2007; 2012; Spisak, Dekker et al., 2012; Spisak, Homan et al., 2012). 그 이유는 지배성이라는 특성은 리더에게 긍정적인 특성으로 평가되기도 하지만, 상대방의 복종성을 수반하 는 개념이라는 측면에서 부정적인 특성으로도 평가되기 때문이다.

Little과 그의 동료들(2007)은 외모적 지배성을 집단의 동의보다는 집단의 순종과 복종에 기반을 두는 리더십 특성으로 평가한다. 실제로, 남성적 외모를 가진 사람은 지배적이면서도, 신뢰할 수 없 는 사람으로 인식되는 경향이 있다(Oosterhof \& Todorov, 2008; Penton-Voak et al., 1999; Perrett et al., 1998). 이는 외모적 지배성의 효과가 상황에 따라 긍정적일 수도 있고 부정적일 수도 있음을 시사한다. 선행 연구들에 따르면, 지배적인 외모의 효과는 상황에 따라 다른 것으로 나타난다. 선행 연구들에서는 진화심리학적 프레임에 근거해서 '상황'을 전시 상황과 평시 상황으로 나눈 다음 피 험자들에게 각각의 상황을 고려해서 외모에 대한 평가를 하도록 하였다(Little, 2014; Little et al., 2007; 2012; Spisak, Dekker et al., 2012; Spisak, Homan et al., 2012). 예를 들어, Little과 그의 동료 들(2007)은 선행연구(Perrett et al., 1998)에서 사용된 기법을 사용하여 남성성이 강한 얼굴과 여성 성이 강한 얼굴을 제작한 후, 두 얼굴을 동시에 피험자들에게 제시했다. 이후, 피험자들에게 본인의 국가를 이끌어갈 사람으로서 누구에게 투표할 것인지에 대해 물었다. 그리고 이어서 전시 상황과 평시 상황에서는 누구에게 투표할 것인지에 대해 추가로 응답하도록 하였다. 연구 결과, 전시 혹은 평시 상황에 대한 고려 없이 투표했을 때는 얼굴에 대한 선호도의 차이가 나타나지 않았으나(남성 성이 강한 얼굴: $51 \%$, 여성성이 강한 얼굴: $49 \%$ ), 전시 상황을 고려했을 때는 통계적으로 유의미하 게 남성성이 강한 얼굴을 더 선호했으며(64\%), 반대로 평시 상황을 고려했을 때는 여성성이 강한 얼굴을 더 선호했다(60\%).

\section{3 신뢰성(trustworthiness)}

기본적으로 리더와 부하 혹은 리더와 동료 간 신뢰는 리더십 발휘에 있어 중요한 요소로 평가된 다(Burke et al., 2007; Dirks \& Ferrin, 2002). 또한, 타인에게 신뢰를 느낀다는 것은 그 대상이 능력 이 있음은 물론, 도덕성과 자비심을 갖추고 있는 사람으로 인식하는 것이다(Little, 2014). 
비록, 외모적 신뢰성이 정치적 선거 결과를 예측하지 못한다는 연구도 있었지만(Todorov et al., 2005), 이와 상반되게 외모적 신뢰성이 리더 선택을 예측한다는 연구 결과도 있었다. 예를 들어, Rule과 그의 동료들의 연구(2010)에서 외모적 신뢰성과 호감도의 평균값(Warmth 요인)이 일본 정 치인들의 선거 결과를 예측하는 것으로 나타났다. 또한, Chen과 그의 동료들(2014)은 외모적 유능 함이 리더에게 가장 우선적으로 기대되는 특성이라는 점에 초점을 두고, 외모적 신뢰성의 효과는 그 대상이 일정 수준 이상의 외모적 유능함의 특성을 갖추고 있을 때에만 나타날 것이라는 가설을 세우고 연구를 진행하였다. 연구 결과, 외모적 신뢰성은 2008년 상원의원 선거에 출마한 후보자들 의 실제 당선 여부를 예측했는데, 이 효과는 후보자들이 외모적으로 유능하다고 판단될 때만 나타 났다(Chen et al., 2014). 또한, 회사의 부당경영 이후, 후임자로서 $\mathrm{CEO}$ 를 선택하는 상황에서는 신 뢰성이 높아 보이는 외모를 가진 사람을 선호하는 것으로 나타나기도 했다(Gomulya et al., 2017).

한편, 외모적 신뢰성은 기본적으로 외모적 남성성과는 부적 관계를 보이는 데 반해, 외모적 여성 성과는 정적 관계를 보이는 외모적 특성으로 알려져 있다(Penton-Voak et al., 1999; Perrett et al., 1998). 이는 평시 상황에 여성적 외모가 더 선호되는 경향성(Little et al., 2007; Spisak, Dekker et al., 2012; Spisak, Homan et al., 2012)이 외모적 신뢰성에서도 나타날 수 있음을 시사한다. 실제로 신뢰가 가는 외모가 전시 상황보다 평시 상황에 더 선호되는 것으로 나타났다(Little et al., 2012).

\section{4 매력(attractiveness)}

매력적인 외모를 가진 사람들도 리더로 더 선호되거나 선택되는 경향이 있다(Banducci et al., 2008; Berggren et al., 2010; Efran \& Patterson, 1974; Langlois et al., 2000; Little et al., 2012; Surawski \& Ossoff, 2006; White et al., 2013). 예를 들어, 10,011명의 사람들이 1,929명의 핀란드 정치적 선거 후보자들의 사진에 대해 평가한 설문을 분석한 연구에 따르면, 외모가 매력적이라고 평가받은 후보자일수록 실제 선거에서 표를 더 많이 받은 것으로 나타났다(Berggren et al., 2010).

기본적으로 매력적인 사람은 행복하고 성공적인 삶을 영위하는 긍정적인 특성을 지닌 사람으로 인식되는 경향이 있다(Dion et al., 1972). 즉, 매력적인 사람은 더 좋은 직업을 가지고, 더 행복한 결혼생활을 하며, 개인적 성취를 더 잘 이룰 것으로 생각된다. 이는 매력의 후광효과라 할 수 있는 데, 사람의 매력을 나타내 주는 가장 대표적인 요소가 그 대상의 외모라는 측면은 매력적인 외모가 리더 선택에 영향을 주는 이유를 뒷받침한다. 예를 들어, 외모적 매력은 리더십 유능성의 특성과 정적인 상관관계가 있는 것으로 나타난다(Surawski \& Ossoff, 2006). 특히, 진화심리학적 프레임에 서는 매력적 외모는 리더의 신체적 건강함을 나타내 주는 단서인 것으로 제시된다(Little et al., 2012; Van Vugt \& Grabo, 2015). 즉, 먼 과거부터 신체적으로 건강한 사람이 그렇지 않은 사람보다 리더로서의 자격을 더 잘 갖춘 사람으로 평가되었을 것이며, 따라서 신체적으로 건강할 것으로 느 껴지는 외모를 가진 사람에게 우리는 매력을 느끼게 되었을 것이라는 것이다. 실제로 늙어 보이는 
외모일수록 덜 매력적인 것으로 평가된다(Todorov et al., 2015).

또한, 리더의 신체적 건강은 상황에 따라 그 중요도가 달라질 수 있는데, 예를 들어 평시 상황보다는 전시 상황일 때 더 중요할 것으로 가정된다(Little et al., 2012). 연구에 따르면, 평시 상황일 때는 신뢰 가 가는 외모를 가진 리더에 대한 선호도가 높은 반면, 전시 상황일 때는 매력적인 외모를 가진 리더에 대한 선호도가 높았다(Little et al., 2012). 또한, White와 그의 동료들(2013)은 이와 관련된 흥미로운 연구 결과를 제시했다. 즉, 질병 위험이 높은 지역일수록 매력적인 외모를 가진 후보자들이 당선될 확 률이 높았으며(2010년도 미국 의회 선거에 출마한 후보자들의 사진을 사용), 혐오스러운 사건에 대한 글을 읽은 집단이 그렇지 않은 집단에 비해 매력적인 외모를 가진 후보자에게 투표하려는 경향성이 더 강한 것으로 나타났다(White et al., 2013). 이는 질병을 회피하려는 사람들의 내재화된 속성이 신체 적 건강을 암시하는 매력적인 외모를 가진 리더에 대한 선호도에 영향을 미친다는 것을 시사한다.

\section{V. 영향 변수}

지금까지 리더 외모의 효과에 대해 밝힌 연구들의 결과를 외모적 유능함, 지배성, 신뢰성, 그리고 매력 4가지의 차원으로 살펴보았다. 한편, 선행 연구들은 리더 외모의 효과가 여러 가지 변수에 의 해 영향을 받는다는 점을 제시하고 있는데, 지금부터 그 변수들에 대해 논의하고자 한다.

첫째, 리더 선택을 실시하는 사람들이 가지고 있는 리더(후보자)에 관한 정보의 양이다. 사람들 은 대상과의 심리적 거리가 멀거나, 대상에 대해 평가할 수 있는 정보가 부족할 때, 더 대상의 외모 에 의존하여 판단하는 경향성이 있다(Chang et al., 2010; Rezlescu et al., 2012). 이는 대상에 관한 정보를 많이 가진 사람들일수록 외모의 영향을 덜 받을 것임을 시사한다. 실제로, 리더 후보자들에 대해 정보를 많이 가지고 있는 사람일수록 외모의 영향을 덜 받는 것으로 나타났다(Ahler et al., 2017). 또한, TV를 시청하는 사람들 중에서 리더에 대한 정보가 부족한 사람들이 특히 대상의 외모 를 기반으로 투표를 하는 것으로 나타났다(Lenz \& Lawson, 2011). 이는 리더로서의 자질을 평가할 수 있는 다양한 정보를 바탕으로 하향식의 리더 선발 체계를 도입하고 있는 기업 및 공공 기관에서 는 리더 외모의 효과가 비교적 적게 나타날 수 있음을 시사한다.

둘째, 동 - 서양의 문화가 리더 외모의 효과에 영향을 미치는 것으로 나타난다(Harms et al., 2012; Na et al., 2015; Rule et al., 2010; Rule et al., 2011). Rule과 그의 동료들의 연구(2010)에 따르면, 일 본인과 미국인들은 일본 및 미국 정치인들의 사진을 통해 대상의 특성을 비슷하게 추론하는 것으로 나타났지만, 선거 결과를 예측하는 외모적 특성은 달랐다. 미국 정치인들의 경우에는, 일본인과 미국 인 피험자가 평가한 Power 합성 점수(지배성과 외모적 성숙성 점수의 평균)가 선거 결과를 유의미 하게 예측한 반면, 일본 정치인들의 경우에는 반대로 Warmth 합성 점수(호감도와 신뢰성 점수의 평 균)가 예측하는 것으로 나타났다. 국내 국회의원 후보자들의 사진을 사용한 연구에서도, 피험자들이 
평가한 국내 정치인들의 외모적 유능함보다 미국 정치인들의 외모적 유능함이 선거 결과를 더 잘 예측하는 것으로 나타났다(Na et al., 2015). 이는 리더의 외모를 통해 추론해 내는 경향성은 문화와 관계없이 동일하게 나타나지만, 그 효과는 문화의 영향을 받는다는 것을 시사한다.

셋째, 집단이 처한 상황이 리더 외모 효과에 영향을 미칠 수 있다. 앞서 언급한 바와 같이, 외모 적 지배성과 신뢰성의 효과가 전 - 평시의 상황에 따라 달라질 수 있다(Little, 2014; Little et al., 2012). 이 외에도 기업의 처한 상황에 따라 외모의 효과성이 달라진다는 연구도 있다. 예를 들어, 회사의 부당경영(misconduct) 이후, 후임자로서 $\mathrm{CEO}$ 를 선택하는 상황에서는 신뢰성이 높아 보이 는 외모를 가진 사람을 선호하는 것으로 나타나기도 했다(Gomulya et al., 2017).

넷째, 리더가 이끄는 집단의 특성 차이의 영향이 있을 수 있다. 일반적으로 정치인들의 경우에는 외모적 유능함이 선거 결과를 잘 예측하는 것으로 나타나는데 반해(Olivola \& Todorov, 2010), 군인 들의 경우에는 외모적 지배성이 그들의 직책 혹은 계급을 잘 예측하는 것으로 나타난다(Mazur et al., 1984; Mueller \& Mazur, 1996; 1997). 또한, 로펌 회사의 관리자들의 경우에는 외모적 지배성과 성숙 성의 평균값(Power 요인)이 그들의 회사 위계질서 상의 지위(leadership rank)를 예측한 반면, 마피아 들의 경우에는 외모적 호감도, 신뢰성, 사회적 유능함의 평균값(Social skill 요인)이 그들의 지위를 예 측했다(Re \& Rule, 2017). 특히, 1996년부터 2006년 사이의 미국 주지사들과 2007년부터 2012년 사이 의 미군 4성 장군 및 2012년도의 1성, 2성, 3성 장군들의 사진을 사용한 연구에서, 정치인들의 외모는 유능해 보이는 경향이 있는데 반해, 군인 장성들은 남성적이고, 성숙하며, 차가워 보이는 경향이 있는 것으로 나타났다(Olivola, Eubanks, \& Lovelace, 2014). 또한, Fortune 500 회사의 CEO 150명과 미국 시민 150 명, 그리고 대학 교수 150 명의 사진을 비교한 연구에서 $\mathrm{CEO}$ 들의 외모는 시민 및 대학 교수 들과 분명히 다른 것으로 나타났다(Stoker et al., 2016). 이는 집단의 특성에 따라 선호되는 외모적 특성이 다르며, 그에 따라 리더 외모의 효과가 달라질 수 있음을 시사한다. 이 외에도 성 편향(Chiao et al., 2008), 정치적 성향(Olivola et al., 2012), 리더의 핵심적 자기평가(core self-evaluation, CSE; Dietl et al., 2018) 등이 리더 외모 효과에 영향을 미치는 변수로 나타나고 있다.

\section{VI. 기존 연구의 한계}

지금까지 최근 15 년 간 활발하게 연구되고 있는 '리더의 외모'에 관한 선행 연구들을 바탕으로, 외모 효과의 이유, 연구 방법, 연구 결과 및 영향 요인을 논의했다. 그러나 대부분의 연구들이 그러 하듯, 리더 외모 연구에도 여러 한계점이 있다. 현재까지 지적되고 있는 연구의 한계점 및 한계점과 관련된 향후 연구방향을 제시하고자 한다.

첫째, 대부분의 리더 외모 연구들이 실험연구보다는 상관연구 설계로 진행됨에 따라, 리더 외모 와 결과변수(리더 선택)의 인과관계를 밝히기보다는 상관관계를 밝히는 데 중점을 두었다는 측면 
이다. 다시 말해, 특정한 외모적 특성을 지닌 리더와 조직의 성과와의 긍정적인 상관관계는 외모적 특성이 조직의 성과를 높인다는 인과적 관계를 상정할 수 없다는 측면이 한계로 지적된다. 따라서, 리더 외모와 그 결과 변수들의 인과성이 주요한 한계점으로 지적된다(Antonakis \& Eubanks, 2017; Poutvaara, 2014). 실험연구 설계를 통해 실시된 연구들도 일부 보고되고 있으나(Fruhen et al., 2015; Little et al., 2007), 리더 외모 효과를 밝히는 다양한 실험연구가 필요할 것으로 보인다.

둘째, 리더 외모를 통한 특성 판단은 주로 피험자들의 주관적인 평가에 의해 실시되었다. 그러나 피험자들의 주관적 평가는 편향적이고 부정확하기 때문에, 보다 명확하고 객관적인 방법을 통한 리 더 외모 평가가 필요하다는 주장도 있다(Stoker et al., 2016). 예를 들어, Olivola, Eubanks와 그의 동료들(2014)은 경영, 군사, 정치 그리고 스포츠 분야 리더들의 사진을 피험자들에게 제시한 후, 그 들의 성과가 얼마나 좋을지에 대해 추정하게 하였는데, 그 결과 피험자들의 추정과 실제 리더들의 성과는 전혀 관계가 없는 것으로 나타났다. 리더 외모에 대한 보다 객관적 평가 지표로서, 얼굴의 너비 대 높이 비율(Alrajih \& Ward, 2014; Lewis et al., 2012; Wong et al., 2011), 혹은 입의 너비 (Re \& Rule, 2016b) 등의 변수가 사용된 바 있다. 비교적 객관적인 외모적 특성을 판단할 수 있는 변수들에 대해 향후 지속적으로 연구될 필요가 있다.

셋째, 리더 외모 연구들이 미국, 캐나다, 그리고 영국 등 서양 문화권에서 주로 이루어지다 보니, 연구에 사용되는 얼굴 자극들이 대부분 서양인의 얼굴이다. 앞서 서술한 바와 같이, 외모를 통한 특 성 추론은 동 - 서양 문화의 영향을 받지 않는 것으로 제시되고 있으나(Rule et al., 2010; 2011), 페이 시즘의 문화적 일치와 관련된 부분은 더 많은 연구를 통해 확인되어야 할 필요성이 있다. 특히, 비교 문화 연구의 프레임을 통해 리더 외모의 효과를 확인한 연구들은 상이한 결과들이 보고되기도 했다 (Re \& Rule, 2015; Rule et al., 2010; 2011). 따라서 리더 외모 효과에 대한 문화의 영향에 대해서 더 많은 연구가 필요하며, 이를 위해서는 특히 동양 문화권에서의 리더 외모 연구가 많이 필요하다.

\section{VII. 결론 및 제언}

리더 외모에 관한 연구가 지속적으로 실시되고 있음에도 불구하고, 리더십 측면에서 리더 외모 효과가 가지는 함의점에 대해서는 여전히 의문시되는 측면이 있다. 예를 들면, 리더 외모 효과는 사람들의 인지적 편향의 결과일 뿐이라는 주장이다(Van Vugt \& Ahuja, 2010; Lenz \& Lawson, 2011; Little \& Roberts, 2012; Olivola, Funk et al., 2014). '리더 외모 효과의 이유' 부분에서 논의한 바와 같이, 인류의 진화 과정에서 리더의 외모를 기반으로 하는 리더에 관한 인지적 원형이 형성되 어 사람들의 뇌에 각인됨에 따라, 현대 사회에서도 여전히 인지적 원형이 리더 선택 과정에 영향을 미치고 있다는 본다. 그리고 이러한 원형은 과거에는 적합했을지라도, 현 시점에서는 적합하지 않 으므로, 인지적 편향의 요소로써 작용하고 있다고 주장한다. 그러나 리더 외모의 효과가 사람들의 
인지적 편향의 결과라고 하더라도, 사람들이 리더를 선호하거나 선택하는 과정에서 여전히 외모가 영향을 미치고 있다는 사실이 중요하다. 따라서 리더와 조직의 입장에서는 사람들의 인지적 편향의 영향이 최소화되도록 제도적 장치를 마련하는 것도 중요하겠지만, 사람들의 이러한 인지적 편향에 대한 충분한 이해를 바탕으로 리더십을 발휘할 필요가 있다.

특히, Shamir(2007)은 리더십 연구가 그동안 리더의 관점에서 주로 연구되어 왔으나, 부하의 관 점을 이해하는 것이 리더십을 이해하는 데 매우 필수적이라는 점을 지적한 바 있다. 암묵적 리더십 이론(Implicit Leadership Theory, ILT; Lord \& Maher, 1993)은 부하의 관점에 기반을 두는 대표적 이론이다(Junker \& Van Dick, 2014). 암묵적 리더십 이론에 의하면, 사람들은 각자 '리더는 이러한 특성을 지니고 있어야 한다 등의 방식으로, 자신만의 독특한 암묵적 리더십 혹은 리더 원형을 가지 고 있다(Lord \& Maher, 1993). ILT는 부하가 리더를 인식하는 과정(Weiss \& Adler, 1981) 및 부하 와 리더의 관계 형성에 영향을 주는 중요한 변수로 평가된다(Epitropaki \& Martin, 2004). 예를 들 어, 부하들의 ILT에 부합하는 리더들은 부하들로부터 더 존경받고(Van Quaquebeke et al., 2011), 더 선호되며(Nye \& Forsyth, 1991), 더 변혁적 리더로 인식되는 경향이 있다(Bass \& Avolio, 1989). 또한, 부하들은 리더가 자신의 ILT에 부합할수록, 직무 만족도가 높고 더 행복한 것으로 나 타난다(Epitropaki \& Martin, 2005). 이는 부하들이 가지고 있는 ILT가 인지적으로 편향되었는지 여부와 관계없이 리더들은 부하들의 ILT를 충분히 이해해야 함을 시사한다. 이러한 점을 반영하듯, Calder(1977)은 '리더십을 가르치는 것은 타인의 인식에 민감해지도록 하는 것이다' 라고 주장하였 으며, Forsyth와 Nye(2008)는 리더십은 단지 리더의 자질이나 행동을 통해서만 이해되어서는 안 되고, 부하들의 인식을 포함하여 이해되어야 한다고 역설했다.

종합하면, 리더 외모와 리더 선택(선호) 관계는 단순히 인지적 편향을 반영하는 것이 아니라, 리 더십을 발휘하는 과정에서 반드시 이해하고 고려해야 할 중요한 요소이다. 그렇다면, 리더 외모 효 과는 군 리더십 발휘에 어떻게 적용될 수 있을까? 첫째, 앞서 기술한 바와 같이 사람들은 죽음을 인식하는 조건과 전시 상황에서 카리스마적이고(Cohen et al., 2004), 신체적으로 건강해 보이는 리 더를 선호한다(Little et al., 2012). 그리고 이러한 상황에서 사람들은 카리스마적 특성을 나타내는 지배적이고 남성적인 외모를 가진 리더(Little, 2014), 그리고 신체적 건강함을 나타내는 매력적인 외모를 가진 리더를 선호한다(Little et al., 2012; White et al., 2013). 이는 군 리더들이 죽음이 지속 적으로 부각되는 전시 상황에서 부하들이 가지게 될 ILT가 카리스마와 신체적 건강함을 내포할 수 있음을 인식하고, 그에 부합하는 형태의 리더십을 발휘할 필요성을 시사한다. 또한, 군 리더들은 말 과 행동을 통해 상황에 부합하는 리더십을 발휘함과 동시에, 이와 부합하는 외모적 특성도 부하들 에게 인식시킬 필요가 있다. 특히, 부하들과 직접적인 상호작용이 불가한 최상위 직책의 리더들의 경우에는 외모적 특성의 효과가 더욱 강하게 나타날 수 있다. 따라서 군 리더들은 전시 상황에 부 하들이 가지게 될 ILT에 부합하는 즉, 부하들이 선호하게 되는 외모적 특성을 효과적으로 드러낼 수 있는 방안은 고려할 필요가 있다.9) 
둘째, 리더 외모 연구는 군 조직에서 실시되고 있는 군 리더에 대한 진급 심사 등의 다양한 인사 평가 과정에 군 리더들의 외모가 영향을 미칠 수 있음을 시사한다. 예를 들면, 앞서 기술한 바와 같이, Olivola, Eubanks와 동료들의 연구(2014)에서 군인 장성들은 남성적이고, 성숙하며, 차가워 보이는 외모를 가진 것으로 나타났다. 물론, 이들이 이러한 외모적 특성을 가졌기 때문에 장성으로 진급을 했는지, 반대로 장성으로 진급해서 이러한 외모적 특성을 가지게 되었는지는 명확하지 않 다. 따라서 군 인사평가 과정에서 외모 효과가 나타나는지, 효과가 나타난다면 어떠한 외모적 특성 이 리더 선택에 영향을 미치는지 등에 관해 실증적인 연구를 실시할 필요가 있다. 또한, 인사평가 과정에 외모의 영향이 있는 것으로 밝혀진다면, 외모 효과를 어떻게 줄일 수 있는지 등에 대해서도 연구될 필요가 있다. 일례로, 미 해군과 공군은 각각 2016년과 1995년을 기점으로 진급 심사 과정에 서 진급 대상자들의 사진을 사용하는 것을 중단한 바 있다(Korenman et al., 2019). 반대로, 리더 외모 효과를 군 리더 임명 과정과 같은 실무적 측면에서도 적용해 보는 방안도 있다. 예를 들어, 적과 직접적으로 대치하고 있는 최전방 부대의 지휘관을 임명하는 경우, 후보자들의 리더십 자질을 평가할 수 있는 여러 지표들이 동일한 상황이라면, 더 카리스마적이 건강해 보이는 외모를 가진 사 람을 임명하는 것도 일부 고려할 수 있는 방안으로 보여진다. 물론, 군 리더 외모의 실무적 적용 방안은 반드시 과학적인 연구 결과가 선행되어야 한다.

최근 15년 간, 리더십 분야에서는 리더 외모의 효과성을 밝히는 다양한 연구들이 실시되어 왔다. 본 연구에서는 사람들이 대상의 외모만을 통해 대상의 특성을 추론해 내는 경향성인 페이시즘에 초점을 두고, 그동안 진행되어 온 관련 연구들을 리더 외모 효과의 이유, 연구방법, 주요 연구결과, 한계점 및 향후 연구방향, 그리고 군 리더십에서의 함의점을 체계적으로 논의하였다. 특히, 전장 상 황에서 남성적, 지배적, 그리고 매력적인 외모가 선호된다는 연구결과는 군 리더의 외모가 군 리더 십 연구 분야에 있어서 충분히 연구가치가 있음을 나타내고 있다. 하지만, 군 리더십 분야에서 페이 시즘에 관한 연구는 아직 시작 단계이며, 가야할 길이 멀다. 본 연구를 통해 페이시즘에 관한 군 리더십 연구자들의 관심을 기대한다.

\section{Acknowledgements}

We would like to thank Editage (www.editage.co.kr) for English language editing.

\section{Declaration of Conflicting Interests}

The author(s) declared no potential conflicts of interest with respect to the research, authorship, and/or publication of this article.

9) Little과 Roberts(2014)는 리더 외모에 대한 연구를 개관하며, 직업적 성공(occupational success)의 관점에서 취업 및 승진 심사자(selector) 및 대상자(candidate)들에게 참고할만한 지침을 제안하기도 했다. 


\section{참고문헌}

Ahler, D. J., Citrin, J., Dougal, M. C., \& Lenz, G. S. (2017). Face value? Experimental evidence that candidate appearance influences electoral choice. Political Behavior, 39(1), 77-102. https://doi.org/10.1007/s11109-016-9348-6

Alrajih, S., \& Ward, J. (2014). Increased facial width-to-height ratio and perceived dominance in the faces of the UK's leading business leaders. British Journal of Psychology, 105(2), 153-161. https://doi.org/10.1111/bjop.12035

Anderson, R. C., \& Klofstad, C. A. (2012). Preference for leaders with masculine voices holds in the case of feminine leadership roles. PloS One, 7(12), e51216. https://doi.org/10.1371/journal.pone.0051216

Antonakis, J., \& Dalgas, O. (2009). Predicting elections: Child's play!. Science, 323(5918), 1183-1183. https://doi.org/10.1126/science.1167748

Antonakis, J., \& Eubanks, D. L. (2017). Looking leadership in the face. Current Directions in Psychological Science, 26(3), 270-275. https://doi.org/10.1177/0963721417705888

Armstrong, J. S., Green, K. C., Jones Jr, R. J., \& Wright, M. J. (2010). Predicting elections from politicians' faces. International Journal of Public Opinion Research, 22(4), 511-522. https://doi.org/10.1093/ijpor/edq038

Ballew, C. C., \& Todorov, A. (2007). Predicting political elections from rapid and unreflective face judgments. Proceedings of the National Academy of Sciences, USA, 104(46), 17948-17953. https://doi.org/10.1073/pnas.0705435104

Banducci, S. A., Karp, J. A., Thrasher, M., \& Rallings, C. (2008). Ballot photographs as cues in low-information elections. Political Psychology, 29(6), 903-917. https://doi.org/10.1111/j.1467-9221.2008.00672.x

Bass, B. M., \& Avolio, B. J. (1989). Potential biases in leadership measures: How prototypes, leniency, and general satisfaction relate to ratings and rankings of transformational and transactional leadership constructs. Educational and Psychological Measurement, 49(3), 509-527. https://doi.org/10.1177/001316448904900302

Bastardoz, N., \& Van Vugt, M. (2019). The nature of followership: Evolutionary analysis and revi ew. The Leadership Quarterly, 30(1), 81-95. https://doi.org/10.1016/j.leaqua.2018.09.004

Berggren, N., Jordahl, H., \& Poutvaara, P. (2010). The looks of a winner: Beauty and electoral success. Journal of Public Economics, 94(1), 8-15. https://doi.org/10.1016/j.jpubeco.2009.1 1.002 
Bjornsdottir, R. T., \& Rule, N. O. (2017). The visibility of social class from facial cues. Journal of Personality and Social Psychology, 113(4), 530-546. https://doi.org/10.1037/pspa0000091

Buckley, F., Collins, N., \& Reidy, T. (2007). Ballot paper photographs and low-information electio ns in Ireland. Politics, 27(3), 174-181. https://doi.org/10.1111/j.1467-9256.2007.00297.x

Burke, C. S., Sims, D. E., Lazzara, E. H., \& Salas, E. (2007). Trust in leadership: A multi-level review and integration. The leadership Quarterly, 18(6), 606-632. https://doi.org/10.1016/j.leaqua.2007.09.006

Calder, B. J. (1977). An attribution theory of leadership. In B. M. Staw \& G. R. Salancik (Eds.), New directions in organizational behavior (pp. 179-204). Chicago: St. Clair Press.

Castelli, L., Carraro, L., Ghitti, C., \& Pastore, M. (2009). The effects of perceived competence and sociability on electoral outcomes. Journal of Experimental Social Psychology, 45(5), 1152-1155. https://doi.org/10.1016/j.jesp.2009.06.018

Chang, L. J., Doll, B. B., van't Wout, M., Frank, M. J., \& Sanfey, A. G. (2010). Seeing is believing: Trustworthiness as a dynamic belief. Cognitive Psychology, 61(2), 87-105. https://doi.org/10.1016/j.cogpsych.2010.03.001

Chen, F. F., Jing, Y., \& Lee, J. M. (2014). The looks of a leader: Competent and trustworthy, but not dominant. Journal of Experimental Social Psychology, 51, 27-33. https://doi.org/10.1016/j.jesp.2013.10.008

Chiao, J. Y., Bowman, N. E., \& Gill, H. (2008). The political gender gap: Gender bias in facial inferences that predict voting behavior. PloS One, 3(10), e3666. https://doi.org/10.1371/jou rnal.pone.0003666

Cogsdill, E. J., Todorov, A. T., Spelke, E. S., \& Banaji, M. R. (2014). Inferring character from faces: A developmental study. Psychological Science, 25(5), 1132-1139. https://doi.org/10. 1177/0956797614523297

Cohen, F., Solomon, S., Maxfield, M., Pyszczynski, T., \& Greenberg, J. (2004). Fatal attraction: The effects of mortality salience on evaluations of charismatic, task-oriented, and relation ship-oriented leaders. Psychological Science, 15(12), 846-851. https://doi.org/10.1111/j.095 6-7976.2004.00765.x

Daros, A. R., Ruocco, A. C., \& Rule, N. O. (2016). Identifying mental disorder from the faces of women with borderline personality disorder. Journal of Nonverbal Behavior, 40(4), 255-281. https://doi.org/10.1007/s10919-016-0237-9

Dietl, E., Rule, N., \& Blickle, G. (2018). Core self-evaluations mediate the association between leaders' facial appearance and their professional success: Adults' and children's perceptio ns. The Leadership Quarterly, 29(4), 476-488. https://doi.org/10.1016/j.leaqua.2018.01.002 
Dion, K., Berscheid, E., \& Walster, E. (1972). What is beautiful is good. Journal of Personality and Social Psychology, 24(3), 285-290. https://doi.org/10.1037/h0033731

Dirks, K. T., \& Ferrin, D. L. (2002). Trust in leadership: Meta-analytic findings and implications for research and practice. Journal of Applied Psychology, 87(4), 611-628. https://doi.org/1 0.1037/0021-9010.87.4.611

Druckman, J. N. (2003). The power of television images: The first Kennedy-Nixon debate revisit ed. The Journal of Politics, 65(2), 559-571. https://doi.org/10.1111/1468-2508.t01-1-00015

Efrain, M. G., \& Patterson, E. W. J. (1974). Voters vote beautiful: the effect of physical appearance on a national election. Canadian Journal of Behavioural Science/Revue canadienne des sciences du comportement, 6(4), 352-356. https://doi.org/10.1037/h0081881

Elgar, M. A. (2016). Leader selection and leadership outcomes: Height and age in a sporting mod el. The Leadership Quarterly, 27(4), 588-601. https://doi.org/10.1016/j.leaqua.2015.12.005

Epitropaki, O., \& Martin, R. (2004). Implicit leadership theories in applied settings: Factor structure, generalizability, and stability over time. Journal of Applied Psychology, 89(2), 293-310. https://doi.org/10.1037/0021-9010.89.2.293

Epitropaki, O., \& Martin, R. (2005). From ideal to real: A longitudinal study of the role of implicit leadership theories on leader-member-exchanges and employee outcomes. Journal of Applied Psychology, 90(4), 659-676. https://doi.org/10.1037/0021-9010.90.4.659

Forsyth, D. R. (2018). Group Dynamics. Mason, OH: Cengage. https://books.google.co.kr/books?h l=ko\&lr=\&id=vg9EDwAAQBAJ\&oi=fnd\&pg=PP1\&dq=Group + dynamics, ${ }^{+}$Forsyth\&ots=t8lt 8TFl40\&sig=7aNUFe4UUk5M0UvT9Yhf8cS7ylQ\&redir_esc $=\mathrm{y} \# \mathrm{v}=$ onepage\&q=Group\%20d ynamics $\% 2 \mathrm{C} \% 20$ Forsyth\&f=false

Forsyth, D. R., \& Nye, J. L. (2008). Seeing and being a leader: The perceptual, cognitive, and interpersonal roots of conferred influence. In C. L. Hoyt, G. R. Goethals, and D. R. Forsyth (Eds.), Leadership at the crossroads: Leadership and psychology (pp. 116-131). Westport, CT: Praeger.

Fruhen, L., Watkins, C. D., \& Jones, B. C. (2015). Perceptions of facial attractiveness, dominance and trustworthiness predict managerial pay awarded in experimental tasks. The Leadership Quarterly, 26(6), 1005-1016. https://doi.org/10.1016/j.leaqua.2015.07.001

George, P. A., \& Hole, G. J. (2000). The role of spatial and surface cues in the age-processing of unfamiliar faces. Visual Cognition, 7(4), 485-509. https://doi.org/10.1080/135062800394621

Gidlow, L. (2014). The great debate: Kennedy, Nixon, and television in the 1960 race for the presidency. The Gilder Lehrman Institute of American History. Retrieved from 
www.gilderlehrman.org/history-byera/sixties/essays/great-debate-kennedy-6ixon-and-t elevision-1960-race-for-presidency

Gomulya, D., Wong, E. M., Ormiston, M. E., \& Boeker, W. (2017). The role of facial appearance on CEO selection after firm misconduct. Journal of Applied Psychology, 102(4), 617-635. https://doi.org/10.1037/apl0000172

Grabo, A., \& van Vugt, M. (2018). Voting for a male warrior or female peacekeeper? Testing the evolutionary contingency hypothesis in the 2016 US presidential elections. Evolutionary Psychology, 16(2), 1-9. https://doi.org/10.1177/1474704918773267

Harms, P. D., Han, G., \& Chen, H. (2012). Recognizing leadership at a distance: A study of leader effectiveness across cultures. Journal of Leadership \& Organizational Studies, 19(2), 164-172. https://doi.org/10.1177/1548051812436812

Hassin, R., \& Trope, Y. (2000). Facing faces: studies on the cognitive aspects of physiognomy. Journal of Personality and Social Psychology, 78(5), 837-852. https://doi.org/10.1037/0022-3514.78.5.837

Junker, N. M., \& Van Dick, R. (2014). Implicit theories in organizational settings: A systematic review and research agenda of implicit leadership and followership theories. The Leadership Quarterly, 25(6), 1154-1173. https://doi.org/10.1016/j.leaqua.2014.09.002

Klofstad, C. A., Anderson, R. C., \& Peters, S. (2012). Sounds like a winner: voice pitch influences perception of leadership capacity in both men and women. Proceedings of the Royal Society B: Biological Sciences, 279(1738), 2698-2704. https://doi.org/10.1098/rspb.2012.0311

Korenman, L. M., Wetzler, E. L., Carroll, M. H., \& Velilla, E. V. (2019). Is it in your face?: Exploring the effects of sexual dimorphism on perception of leadership potential. Military Psychology, 31(2), 107-116.. https://doi.org/10.1080/08995605.2018.1556555

Ku, X. (2019). Faces of Presidents in Africa: Terror attack affects voting decisions in presidential elections in Africa. Unpublished manuscript.

Langlois, J. H., Kalakanis, L., Rubenstein, A. J., Larson, A., Hallam, M., \& Smoot, M. (2000). Maxims or myths of beauty? A meta-analytic and theoretical review. Psychological Bulletin, 126(3), 390-423. https://doi.org/10.1037/0033-2909.126.3.390

Lawson, C., Lenz, G. S., Baker, A., \& Myers, M. (2010). Looking like a winner: Candidate appearance and electoral success in new democracies. World Politics, 62(4), 561-593. https://doi.org/10.1017/S0043887110000195

Lenz, G. S., \& Lawson, C. (2011). Looking the part: Television leads less informed citizens to vote based on candidates' appearance. American Journal of Political Science, 55(3), 574 - 
589. https://doi.org/10.1111/j.1540-5907.2011.00511.x

Lewis, G. J., Lefevre, C. E., \& Bates, T. C. (2012). Facial width-to-height ratio predicts achievement drive in US presidents. Personality and Individual Differences, 52(7), 855-857. https://doi.org/10.1016/j.paid.2011.12.030

Lindqvist, E. (2012). Height and leadership. Review of Economics and Statistics, 94(4), 1191-1196. https://doi.org/10.1162/REST_a_00239

Little, A. C. (2014). Facial appearance and leader choice in different contexts: Evidence for task contingent selection based on implicit and learned face-behaviour/face-ability associations. The Leadership Quarterly, 25(5), 865-874. https://doi.org/10.1016/j.leaqua.2014.04.002

Little, A. C., Burriss, R. P., Jones, B. C., \& Roberts, S. C. (2007). Facial appearance affects voting decisions. Evolution and Human Behavior, 28(1), 18-27. https://doi.org/10.1016/j.evolhumbehav.2006.09.002

Little, A. C., \& Perrett, D. I. (2007). Using composite images to assess accuracy in personality attribution to faces. British Journal of Psychology, 98(1), 111-126. https://doi.org/10.1348/000712606X109648

Little, A. C., \& Roberts, S. C. (2012). Evolution, appearance, and occupational success. Evolutionary Psychology, 10(5), 782-801. https://doi.org/10.1177/147470491201000503

Little, A. C., Roberts, S. C., Jones, B. C., \& DeBruine, L. M. (2012). The perception of attractiveness and trustworthiness in male faces affects hypothetical voting decisions differently in wartime and peacetime scenarios. The Quarterly Journal of Experimental Psychology, 65(10), 2018-2032. https://doi.org/10.1080/17470218.2012.677048

Livingston, R. W., \& Pearce, N. A. (2009). The teddy-bear effect: Does having a baby face benefit black chief executive officers? Psychological Science, 20, 1229 - 1236. https://doi.org/10.1111/j.1467-9280.2009.02431.x

Lord, R. G., \& Maher, K. J. (1993). Leadership and information processing: Linking perceptions and performance. London: Routledge.

Lutz, G. (2010). The electoral success of beauties and beasts. Swiss Political Science Review, 16(3), 457-480. https://doi.org/10.1002/j.1662-6370.2010.tb00437.x

Mazur, A., Mazur, J., \& Keating, C. (1984). Military rank attainment of a West Point class: Effects of cadets' physical features. American Journal of Sociology, 90(1), 125-150. https://doi.org/10.1086/228050

Mueller, U., \& Mazur, A. (1996). Facial dominance of West Point cadets as a predictor of later military rank. Social Forces, 74(3), 823-850. https://doi.org/10.1093/sf/74.3.823 
Muller, U., \& Mazur, A. (1997). Facial dominance in Homo sapiens as honest signaling of male quality. Behavioral Ecology, 8(5), 569-579. https://doi.org/10.1093/beheco/8.5.569

Na, J., Kim, S., Oh, H., Choi, I., \& O’Toole, A. (2015). Competence judgments based on facial appearance are better predictors of American elections than of Korean elections. Psychological Science, 26(7), 1107-1113. https://doi.org/10.1177/0956797615576489

Nana, E., Jackson, B., \& Burch, G. S. J. (2010). Attributing leadership personality and effectiveness from the leader's face: an exploratory study. Leadership \& Organization Development Journal, 31(8), 720-742. https://doi.org/10.1108/01437731011094775

Nye, J. L., \& Forsyth, D. R. (1991). The effects of prototype-based biases on leadership appraisals: A test of leadership categorization theory. Small Group Research, 22(3), 360 379. https://doi.org/10.1177/1046496491223005

Olivola, C. Y, Eubanks, D. L, \& Lovelace, J. B. (2014). The many (distinctive) faces of leadership: inferring leadership domain from facial appearance. The Leadership Quarterly. 25(5), 817-834. https://doi.org/10.1016/j.leaqua.2014.06.002

Olivola, C. Y., Funk, F., \& Todorov, A. (2014). Social attributions from faces bias human choices. Trends in Cognitive Sciences, 18(11), 566-570. https://doi.org/10.1016/j.tics.2014.09.007

Olivola, C. Y., Sussman, A. B., Tsetsos, K., Kang, O. E., \& Todorov, A. (2012). Republicans prefer Republican-looking leaders: Political facial stereotypes predict candidate electoral success among right-leaning voters. Social Psychological and Personality Science, 3(5), 605-613. https://doi.org/10.1177/1948550611432770

Olivola, C. Y., \& Todorov, A. (2010). Elected in 100 milliseconds: Appearance-based trait inferences and voting. Journal of Nonverbal Behavior, 34, $83-110$. https://doi.org/10.1007/s10919-009-0082-1

Oosterhof, N. N., \& Todorov, A. (2008). The functional basis of face evaluation. Proceedings of the National Academy of Sciences, 105(32), 11087-11092. https://doi.org/10.1073/pnas.0805664105

Penton-Voak, I. S., Perrett, D. I., Castles, D. L., Kobayashi, T., Burt, D. M., Murray, L. K., \& Minamisawa, R. (1999). Menstrual cycle alters face preference. Nature, 399, 741-742. https://doi.org/10.1038/21557

Perrett, D. I., Lee, K. J., Penton-Voak, I. S., Rowland, D. R., Yoshikawa, S., Burt, D. M., Henzi, S. P., Castles, D. L., \& Akamatsu, S. (1998). Effects of sexual dimorphism on facial attractiveness. Nature, 394, 884-887. https://doi.org/10.1038/29772

Petersen, M. B., \& Aarøe, L. (2012). Is the political animal politically ignorant? Applying evolutionary psychology to the study of political attitudes. Evolutionary Psychology, 
10(5), 802-817. https://doi.org/10.1177/147470491201000504

Poutvaara, P. (2014). Facial appearance and leadership: An overview and challenges for new research. The Leadership Quarterly, 25(5), 801-804. https://doi.org/10.1016/j.leaqua.2014.08.003

Poutvaara, P., Jordahl, H., \& Berggren, N. (2009). Faces of politicians: Babyfacedness predicts inferred competence but not electoral success. Journal of Experimental Social Psychology, 45(5), 1132-1135. https://doi.org/10.1016/j.jesp.2009.06.007

Re, D. E., DeBruine, L. M., Jones, B. C., \& Perrett, D. I. (2013). Facial cues to perceived height influence leadership choices in simulated war and peace contexts. Evolutionary Psychology, 11(1), 89-103. https://doi.org/10.1177/147470491301100109

Re, D. E., \& Rule, N. O. (2015). CEO facial appearance, firm performance, and financial success. CEO branding: Meaning, measuring, managing, 219-238. http://www2.psych.utoronto.ca/users/rule/pubs/2015/Re\&Rule(2015_CEO).pdf

Re, D. E., \& Rule, N. O. (2016b). The big man has a big mouth: Mouth width correlates with perceived leadership ability and actual leadership performance. Journal of Experimental Social Psychology, 63, 86-93. https://doi.org/10.1016/j.jesp.2015.12.005

Re, D. E., \& Rule, N. O. (2016a). Predicting firm success from the facial appearance of Chief Executive Officers of non-profit organizations. Perception, 45(10), 1137-1150. https://doi.org/10.1177/0301006616652043

Re, D. E., \& Rule, N. O. (2017). Distinctive facial cues predict leadership rank and selection. Personality and Social Psychology Bulletin, 43(9), 1311-1322. https://doi.org/10.1177/0146167217712989

Rezlescu, C., Duchaine, B., Olivola, C. Y., \& Chater, N. (2012). Unfakeable facial configurations affect strategic choices in trust games with or without information about past behavior. PLoS one, 7(3), e34293. https://doi.org/10.1371/journal.pone.0034293

Rosar, U., Klein, M., \& Beckers, T. (2008). The frog pond beauty contest: Physical attractiveness and electoral success of the constituency candidates at the North Rhine Westphalia state election of 2005. European Journal of Political Research, 47(1), 64-79. https://doi.org/10.1111/j.1475-6765.2007.00720.x

Rule, N. O., \& Alaei, R. (2016). "Gaydar" The Perception of Sexual Orientation From Subtle Cues. Current Directions in Psychological Science, 25(6), 444-448. https://doi.org/10.1177/0963721416664403

Rule, N. O., \& Ambady, N. (2008). The face of success: Inferences from Chief Executive Officers' appearance predict company profits. Psychological Science, 19, 109-111. 
https://doi.org/10.1111/j.1467-9280.2008.02054.x

Rule, N. O., \& Ambady, N. (2009). She's got the look: Inferences from female chief executive officers' faces predict their success. Sex Roles, 61, 644-652. https://doi.org/10.1007/s11199-009-9658-9

Rule, N. O., \& Ambady, N. (2010). Democrats and Republicans can be differentiated from their faces. PloS One, 5(1), e8733. https://doi.org/10.1371/journal.pone.0008733

Rule, N. O., Ambady, N., Adams Jr, R. B., Ozono, H., Nakashima, S., Yoshikawa, S., \& Watabe, M. (2010). Polling the face: prediction and consensus across cultures. Journal of Personality and Social Psychology, 98(1), 1-15. https://doi.org/10.1037/a0017673

Rule, N. O., Ishii, K., \& Ambady, N. (2011). Cross-cultural impressions of leaders' faces: Consensus and predictive validity. International Journal of Intercultural Relations, 35(6), 833-841. https://doi.org/10.1016/j.ijintrel.2011.06.001

Sacco, D. F., \& Brown, M. (2018). Preferences for facially communicated big five personality traits and their relation to self-reported big five personality. Personality and Individual Differences, 134, 195-200. https://doi.org/10.1016/j.paid.2018.06.024

Senior, C., Martin, R., Thomas, G., Topakas, A., West, M., \& Yeats, R. (2012). Developmental stability and leadership effectiveness. The Leadership Quarterly, 23, 281-291. https://doi.org/10.1016/j.leaqua.2011.08.005

Shamir, B. (2007). From passive recipients to active co-producers-Followers' roles in the leadership process. In B. Shamir, P. Pillai, M. C. Bligh, \& M. Uhl-Bien (Eds.), Follower-centered perspectives on leadership - A tribute to the memory of James $R$. Meindl (pp. 9-39). Greenwich, CT: Information Age Publishing.

Solomon, S., Greenberg, J., \& Pyszczynski, T. (1991). A terror management theory of social behavior: The psychological functions of self-esteem and cultural world-views. Advances in Experimental Social Psychology, 24, 93-159. https://doi.org/10.1016/S0065-2601(08)60328-7

Spisak, B. R., Dekker, P. H., Krüger, M., \& Van Vugt, M. (2012). Warriors and peacekeepers: Testing a biosocial implicit leadership hypothesis of intergroup relations using masculine and feminine faces. PloS one, 7(1), e30399. https://doi.org/10.1371/journal.pone.0030399

Spisak, B. R., Grabo, A. E., Arvey, R. D., \& Van Vugt, M. (2014). The age of exploration and exploitation: Younger-looking leaders endorsed for change and older-looking leaders endorsed for stability. The Leadership Quarterly, 25(5), 805-816. https://doi.org/10.1016/j.leaqua.2014.06.001

Spisak, B. R., Homan, A. C., Grabo, A., \& Van Vugt, M. (2012). Facing the situation: 
Testing a biosocial contingency model of leadership in intergroup relations using masculine and feminine faces. The Leadership Quarterly, 23(2), 273-280. https://doi.org/10.1016/j.leaqua.2011.08.006

Stoker, J. I., Garretsen, H., \& Spreeuwers, L. J. (2016). The facial appearance of CEOs: Faces signal selection but not performance. PloS One, 11(7), e0159950. https://doi.org/10.1371/journal.pone.0159950

Surawski, M. K., \& Ossoff, E. P. (2006). The effects of physical and vocal attractiveness on impression formation of politicians. Current Psychology, 25(1), 15-27. https://doi.org/10.1007/s12144-006-1013-5

Sussman, A. B., Petkova, K., \& Todorov, A. (2013). Competence ratings in US predict presidential election outcomes in Bulgaria. Journal of Experimental Social Psychology, 49(4), 771-775. https://doi.org/10.1016/j.jesp.2013.02.003

Tigue, C. C., Borak, D. J., O'Connor, J. J., Schandl, C., \& Feinberg, D. R. (2012). Voice pitch influences voting behavior. Evolution and Human Behavior, 33(3), 210-216. https://doi.org/10.1016/j.evolhumbehav.2011.09.004

Todorov, A., Mandisodza, A. N., Goren, A., \& Hall, C. C. (2005). Inferences of competence from faces predict election outcomes. Science, 308(5728), 1623-1626. https://doi.org/10.1126/science.1110589

Todorov, A., Olivola, C. Y., Dotsch, R., \& Mende-Siedlecki, P. (2015). Social attributions from faces: Determinants, consequences, accuracy, and functional significance. Annual Review of Psychology, 66, 519-545. https://doi.org/10.1146/annurev-psych-113011-143831

Todorov, A., Pakrashi, M., \& Oosterhof, N. N. (2009). Evaluating faces on trustworthiness after minimal time exposure. Social Cognition, 27(6), 813-833. https://doi.org/10.1521/soco.2009.27.6.813

Trent, J. S., Mongeau, P. A., Trent, J. D., Kendall, K. E., \& Cushing, R. B. (1993). The ideal candidate: A study of the desired attributes of the public and the media across two presidential campaigns. American Behavioral Scientist, 37(2), 225-239. https://doi.org/10.1177/0002764293037002010

Trichas, S., Schyns, B., Lord, R., \& Hall, R. (2017). "Facing" leaders: Facial expression and leadership perception. The Leadership Quarterly, 28(2), 317-333. https://doi.org/10.1016/j.leaqua.2016.10.013

Tskhay, K. O., \& Rule, N. O. (2016). People automatically extract infants' sex from faces. Journal of Nonverbal Behavior, 40(4), 247-254. https://doi.org/10.1007/s10919-016-0235-y

Van Quaquebeke, N., van Knippenberg, D., \& Brodbeck, F. C. (2011). More than meets the eye: 
The role of subordinates' self-perceptions in leader categorization processes. The Leadership Quarterly, 22(2), 367 - 382. https://doi.org/10.1016/j.leaqua.2011.02.011

Van Vugt, M., \& Ahuja, A. (2010). Selected: Why Some People Lead, Why Others Follow, and Why it Matters. London: Profile Books.

Van Vugt, M., \& Grabo, A. E. (2015). The many faces of leadership: An evolutionary-psychology approach. Current Directions in Psychological Science, 24(6), 484-489. https://doi.org/10.1177/0963721415601971

Vardiman, P. D., Houghton, J. D., \& Jinkerson, D. L. (2006). Environmental leadership development. Leadership \& Organization Development Journal, 27(2), 93-105, https://doi.org/10.1108/01437730610646606

Weiss, H. M., \& Adler, S. (1981). Cognitive complexity and the structure of implicit leadership theories. Journal of Applied Psychology, 66(1), $69-78$. https://doi.org/10.1037/0021-9010.66.1.69

White, A. E., Kenrick, D. T., \& Neuberg, S. L. (2013). Beauty at the ballot box: Disease threats predict preferences for physically attractive leaders. Psychological Science, 24(12), 2429-2436. https://doi.org/10.1177/0956797613493642

Willis, J., \& Todorov, A. (2006). First impressions: Making up your mind after 100 ms exposure to a face. Psychological Science, 17(7), 592-598. https://doi.org/10.1111/j.1467-9280.2006.01750.x

Wong, E. M., Ormiston, M. E., \& Haselhuhn, M. P. (2011). A face only an investor could love: CEOs' facial structure predicts their firms' financial performance. Psychological Science, 22(12), 1478-1483. https://doi.org/10.1177/0956797611418838 


\title{
페이시즘과 군 리더십
}

\author{
구자일* · 현승주 ${ }^{* *}$ · 고재원 ${ }^{* *}$
}

사람들은 대상의 외모를 통해 즉각적으로, 그리고 비교적 정확하게 대상의 심리적 특성을 추론하고 판단하 는 경향성이 있는데 이를 페이시즘(face-ism)이라 한다. 특히, 최근 15년간 리더십 분야에서는 페이시즘과 리 더십(리더 선택 혹은 선호도)의 관련성을 밝히는 연구가 활발히 진행 중이다. 연구 결과를 종합하면, 유능함, 지배성, 신뢰성, 매력 등과 같은 외모적 특성을 지닌 사람들이 그렇지 않은 사람들에 비해 리더로 선호되는 경향성이 있으며, 외모의 효과는 상황(전시 및 평시)에 따라 상이하다는 것이다. 본 연구는 페이시즘과 리더십 의 연구들을 체계적으로 소개하고, 특히 군 리더십에 있어서 가질 수 있는 함의점을 논의하는 데 목적을 두고 실시되었다. 리더 외모가 리더 선택(선호도)에 영향을 미치는 이유, 주된 연구방식, 연구결과, 연구한계 및 향 후 연구방향 및 군 리더십 적용방안 순으로 논의를 진행하였다.

주제어 : 페이시즘, 리더십, 리더 선택, 군 리더십

* (제1저자) 육군사관학교 심리학과, 조교수

** (공동저자) 육군사관학교 심리학과, 강사

*** (교신저자) 육군사관학교 심리학과, 교수 
